\section{Optical bend sensor based on a long-period fiber grating monitored by an optical time-domain reflectometer}

\author{
Orlando Frazão \\ Rua do Campo Alegre, 687 \\ 4169-007 Porto, Portugal \\ Rosane Falate \\ INESC-Porto \\ Rua do Campo Alegre, 687 \\ 4169-007 Porto, Portugal \\ and \\ Centro Federal de Educação \\ Tecnológica do Paraná \\ 80230-901 Curitiba, Brazil \\ Jose M. Baptista \\ INESC-Porto \\ Rua do Campo Alegre, 687 \\ 4169-007 Porto, Portugal \\ and \\ Instituto de Engenharia do Porto \\ Dep. de Engenharia Electrotécnica \\ 4200-072 Porto, Portugal

\section{Jose L. Fabris \\ Centro Federal de Educação \\ Tecnológica do Paraná \\ 80230-901 Curitiba, Brazil} \\ Jose L. Santos, MEMBER SPIE \\ INESC-Porto \\ Rua do Campo Alegre, 687 \\ 4169-007 Porto, Portugal \\ and \\ Universidade do Porto \\ Faculdade de Ciências \\ Departamento de Física \\ Rua do Campo Alegre, 687 \\ 4169-007 Porto, Portugal
}

Instituto de Engenharia de Sistemas e

Computadores do Porto (INESC-Porto)

Rua Dr. António Bernardino de Almeida, 431

\begin{abstract}
We report an alternative technique to interrogate a long-period fiber grating (LPG) when the grating sensitivity is based on the peak amplitude changes of the resonant wavelength. To read the amplitude changes, a conventional optical domain reflectometer was used. Bend measurements were performed to apply such method and to determine the grating sensitivity for this physical parameter. Reflective measurements, temperature insensitive, and the possibility of multiplexing LPG sensors are some advantages offered by this technique. (c) 2005 Society of Photo-Optical Instrumentation Engineers.

[DOI: 10.1117/1.2123267]
\end{abstract}

Subject terms: long-period fiber grating; OTDR; bend sensor.

Paper 050609LR received Jul. 27, 2005; revised manuscript received Aug. 19, 2005; accepted for publication Sep. 21, 2005; published online Nov. 10, 2005.

\section{Introduction}

A long-period fiber grating (LPG) is a periodic refractive index structure on the fiber core whose periodicity is in the range of several hundred micrometers. ${ }^{1}$ The large period of modulation promotes the optical coupling between the propagating core mode and copropagating cladding modes. Since the cladding modes, consequently the resonant peaks, are sensitive to the physical parameters, ${ }^{2}$ curvature or bending measurement can be performed utilizing LPGs. In fact, several authors have demonstrated the bending-induced wavelength shift by means of LPGs. ${ }^{3,4}$ However, the cross sensitivity when different physical parameters are changed at the same time continues to be a common problem of using this kind of grating structure as an optical sensor. Trying to surpass part of this problem, Ye et al..$^{5}$ proposed a sensor head based on LPGs operating at short wavelengths for simultaneous measurement of temperature and bend.

Another problem with the usage of LPGs is the difficulty to interrogate its spectral response since the resonant bands have large bandwidth. Commonly an optical spectrum analyzer (OSA) is used, but this equipment is unpractical for real-time applications due to its size and high cost. Because of this, Allsop et al. ${ }^{6}$ proposed a new method for LPG interrogation based on derivative spectroscopy technique.

In this work, we present an optical bend sensor that uses an LPG written by electric arc discharge and interrogated by a conventional optical time-domain reflectometer (OTDR). The proposed system detects the losses variation of the LPG when the bend is applied and permits a stable measurement independent of temperature fluctuation. A multiplexing experiment using two LPGs in series was also demonstrated.

\section{Experimental Results}

An LPG with a period $\Lambda=540 \mu \mathrm{m}$ and length $L_{L P G}$ $=21.6 \mathrm{~mm}$ was arc-induced in Corning SMF-28 fiber. During the grating inscription, the fiber was kept under a tension of $5.1 \mathrm{~g}$, and was subjected to 40 arc discharges with $9 \mathrm{~mA}$ of current and 1-s duration. With the OSA resolution set to $0.1 \mathrm{~nm}$ and a wavelength range from 1520 to $1570 \mathrm{~nm}$, we first observed the transmission spectrum when the LPG was straight. The obtained central wavelength for this condition was $1557 \mathrm{~nm}$. Second, for the bend tests, a section of fiber $(2 L=330 \mathrm{~mm})$ with a LPG in the middle was clamped between a translation stage (TS) and a fixed base. The sensor curvature $R$ is given by : $R=2 d /\left(d^{2}+L^{2}\right)$, where $d$ is the bending displacement at the center of the LPG and $L$ is the half distance between the edges of the two clampers (see Fig. 1). Figure 2 shows the transmission spectrum of LPG when curvature is applied. We observed that the wavelength and the attenuation of the central peak are changed with the curvature.

To characterize the LPG sensitivity to the applied curvature without the OSA, we used a commercial OTDR to read the losses generated by the LPG (see Fig. 1). In this experience, the optical bend sensor was placed between two optical fiber rolls (SMF 28) with lengths of 3500 and $100 \mathrm{~m}$, respectively. The loss of the LPG sensor is measured using the internal multimode laser of OTDR with

0091-3286/2005/\$22.00 @ 2005 SPIE 


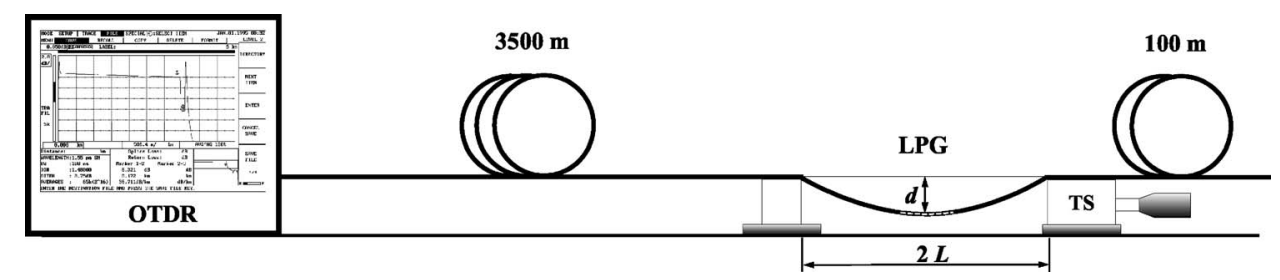

Fig. 1 Experimental setup using an OTDR.

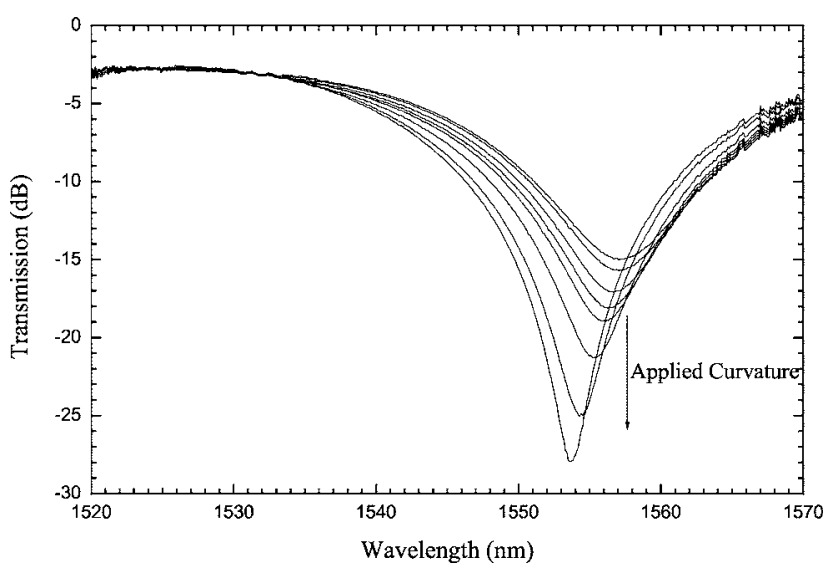

Fig. 2 Bend sensitivity of LPG obtained by an optical spectrum analyzer.

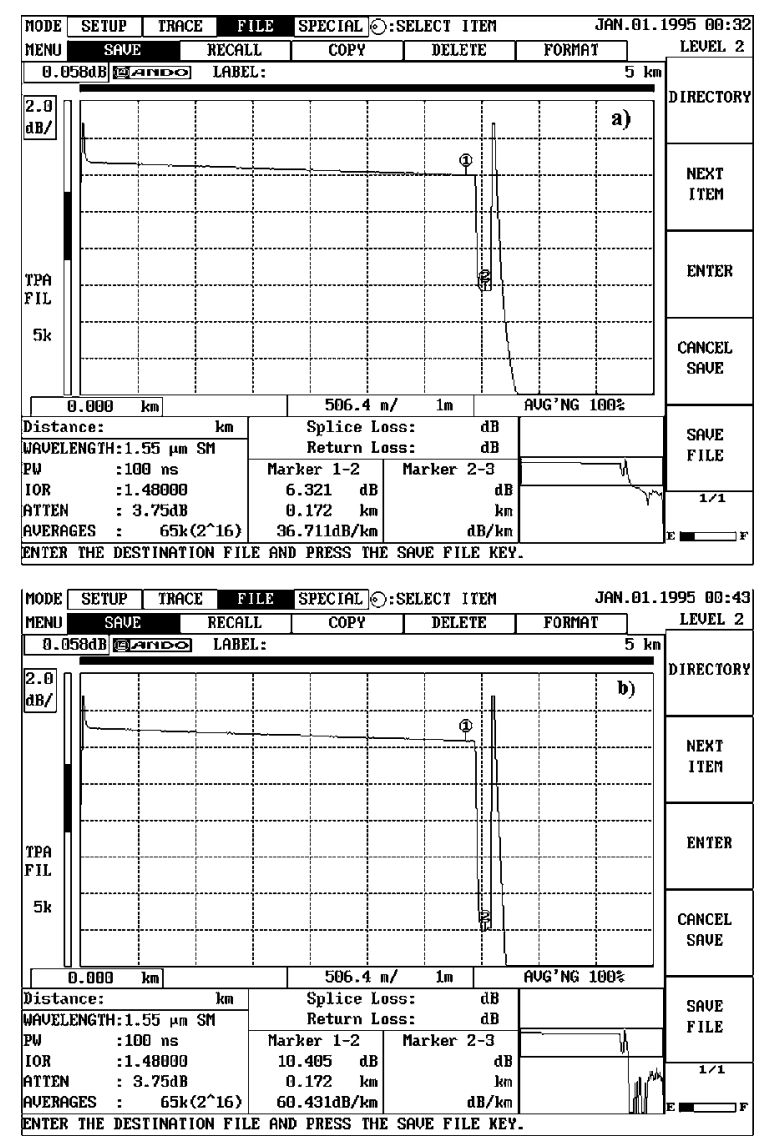

Fig. 3 LPG response observed by the OTDR when curvature is applied for: (a) $R=2.3 \mathrm{~m}^{-1}$ and (b) $R=5.5 \mathrm{~m}^{-1}$.

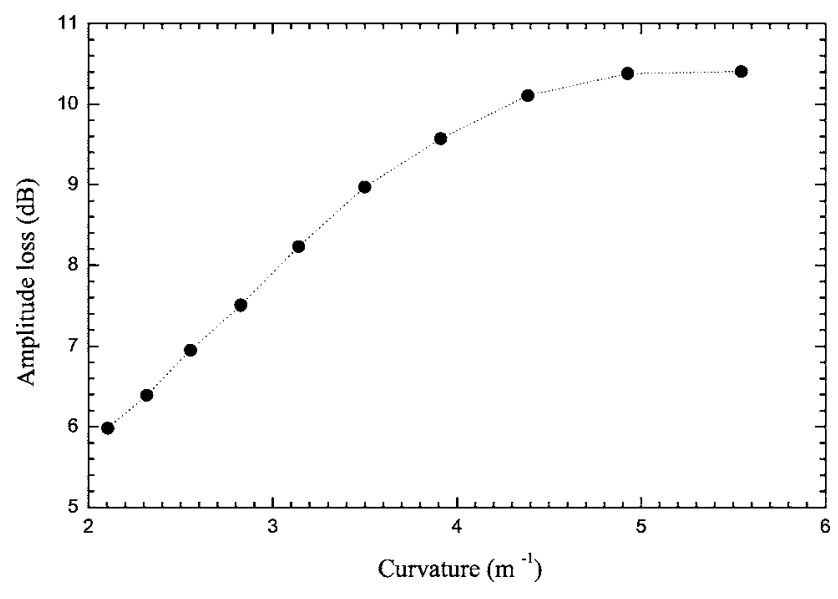

Fig. 4 Evolution of LPG amplitude loss against curvature.

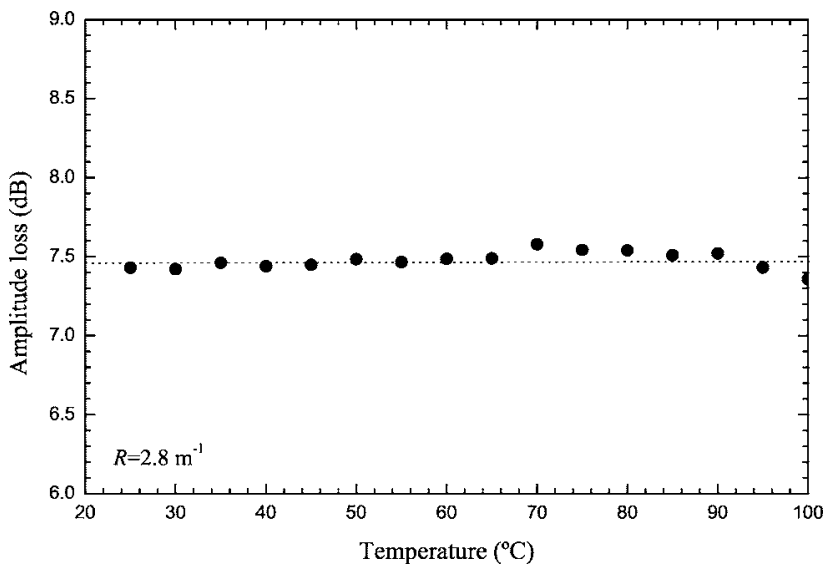

Fig. 5 LPG amplitude loss insensitivity to temperature for a fixed curvature of $2.8 \mathrm{~m}^{-1}$.

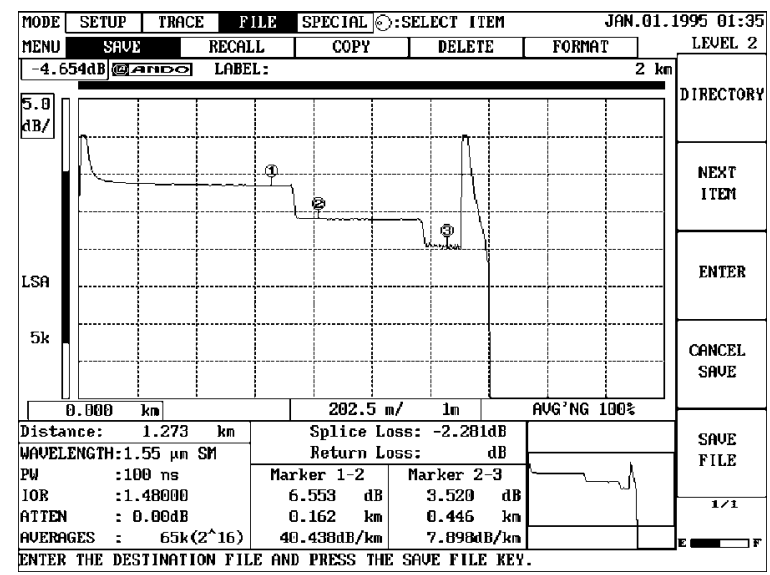

Fig. 6 OTDR measurement when bending two LPGs in series. 
pulses of $100 \mathrm{~ns}$ and working at $1550 \mathrm{~nm}$. When the LPG is illuminated by the laser pulse, the LPG resonance near $1550 \mathrm{~nm}$ increased the backscattering, which was sent in reflection, and was analyzed by the OTDR. Since the peak amplitude loss of the LPG sensor increases when curvature is applied, a higher backscattering is produced at the LPG location that is detected by the OTDR. The opposite amplitude sensitivity of LPG for curvature increment is observed because the LPG is working in the recoupling mode region.

Figure 3 shows the OTDR measurements for two different applied curvatures on the LPG. The valley and the peak at $3500 \mathrm{~m}$ correspond to the loss of the LPG sensor and the Fresnel reflection, respectively. It can be observed that the valley goes deeper as a result of the curvature increments [from Fig. 3(a) to 3(b)] while the fiber backscattering before the LPG is the same, being our reference point. This result shows that when the attenuation peak of LPG increases, the valley attenuation also increases.

Figure 4 presents the relationship between the loss and the curvature of the LPG. The loss values were obtained using two markers given by the OTDR. They are chosen to be located before (reference point) and at the LPG loss. For a curvature range between 2.0 and $5.5 \mathrm{~m}^{-1}$, it obtained an amplitude loss shift of more than $4 \mathrm{~dB}$. Figure 4 also shows two different regions of the LPG response. The first is a linear region, up to $3.5 \mathrm{~m}^{-1}$, with a sensitivity of $2.16 \mathrm{~dB} / \mathrm{m}^{-1}$ and a maximum error of $\pm 0.02 \mathrm{~dB} / \mathrm{m}^{-1}$. The other region shows a loss saturation of the LPG since the LPG reaches the maximum amplitude loss when the curvature is applied. After this loss saturation region, for curvature values higher than $5.5 \mathrm{~m}^{-1}$, the LPG mode energy is recoupled and the amplitude loss decreases. This behavior limits the measurement range to the presented set of curvature measurements. However, such limitation is intrinsic to the LPG sensor. To surpass this, a new LPG design is necessary to increase the curvature range.

To confirm that this technique is temperature insensitive, since the spectral dependence on temperature of the LPG is almost in wavelengths, we inserted the LPG sensor in an oven with a curvature of $2.8 \mathrm{~m}^{-1}$. The LPG was subject to each tested temperature for a period of $15 \mathrm{~min}$, after which the measurements were taken. Figure 5 shows a loss fluctuation of $0.22 \mathrm{~dB}$ for a temperature range between 25 and $100^{\circ} \mathrm{C}$. The small fluctuations do not show any defined slope as a function of the temperature. However, for hightemperature changes, increasing the temperature induces an LPG resonance wavelength change, which detunes its resonance wavelength relative to the wavelength of the internal laser of the OTDR, resulting in loss fluctuations. On the other hand, for low-temperature changes (less than $20^{\circ} \mathrm{C}$ ), these fluctuations will represent less than $0.05 \mathrm{~m}^{-1}$ allowing measure curvature changes higher than this value.

Figure 6 shows the OTDR measurement when multiplexing two LPGs. The LPGs are mounted in series and have similar characteristics, with their peak attenuation small, so that both gratings responses can be monitored by the OTDR. It was observed that each LPG had its own response when different curvature was applied, showing that this system is able to determine different curvatures along a fiber link. Although this multiplexing was demonstrated, it was verified that this setup is limited in the number of serial sensors due to the dynamic range of the OTDR.

\section{Conclusions}

This work presents a simple technique to interrogate LPGs. It consists of utilizing a commercial OTDR to read the LPG loss evolution when the bend or curvature is applied. This interrogation technique is insensitive to the physical parameters that only change the LPG spectral response in wavelengths and has the capability of multiplexing LPG sensors in series. However, a low peak attenuation of the LPGs is necessary since for each LPG inserted in the fiber link an additional loss is measured by the OTDR and the lower limit power measurement of OTDR can be reached.

\section{Acknowledgments}

R. Falate has her work supported in part by Coordenação de Aperfeiçoamento de Pessoal de Nível Superior (CAPES), Grant BEX: 0301/04-3, Brazilian Agency.

\section{References}

1. A. M. Vengsarkar, P. Lemaire, J. B. Judkins, V. Bhatia, T. Erdogan, and J. E. Sipe, "Long-period fiber gratings as band-rejection filters," J. Lightwave Technol. 14(1), 58-64 (1996).

2. V. Bhatia and A. M. Vengsarkar, "Optical fibre long-period grating sensors," Opt. Lett. 21(9), 692-694 (1996).

3. H. J. Patrick, C. C. Chang, and S. T. Vohra, "Long period fibre gratings for structural bend sensing," Electron. Lett. 34(18), 1773-1775 (1998).

4. Y. Liu, L. Zhang, J. A. R. Williams, and I. Bennion, "Optical bend sensors based on measurement of resonance mode splitting of longperiod fiber grating," IEEE Photon. Technol. Lett. 12(5), 531-533 (2000).

5. C. C. Ye, S. W. James, and R. P. Tatam, "Simultaneous temperature and bend sensing with long-period fiber gratings," Opt. Lett. 25(14), 1007-1009 (2000)

6. T. Allsop, T. Earthrowl, R. Reeves, D. J. Webb, and I. Bennion, "The interrogation and multiplexing of long period grating curvature sensors using a Bragg grating based, derivative spectroscopy technique," Meas. Sci. Technol. 15(1), 44-48 (2004).

7. W. Du, H. Tam, M. Liu, and X. Tao, "Long period fiber grating bending sensor in laminates structures," Proc. SPIE 3330, 284-292 (1998). 